\title{
Desarme para el desarrollo humano: De una cultura de guerra a una cultura de Paz y no violencia ${ }^{1}$
}

\section{Resumen}

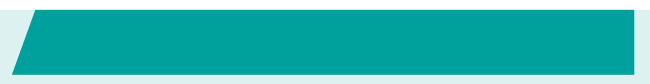

Federico Mayor Zaragoza afirma en el presente texto, que es moralmente inaceptable que cada día mueran de hambre miles y miles de personas cuando se invierten en gastos militares y armamento millones de dólares. Advierte que la mejor solución es el desarme, incluyendo el nuclear y que actualmente, se está llegando a puntos de no retorno en cuestiones esenciales del legado intergeneracional.

Señala lo urgente de un nuevo concepto de seguridad, dado a que los grandes poderes siguen pensando que la fuerza militar es la única expresióny referencia de "seguridad". Las soluciones existen. La acción inmediata es necesaria para cumplir con nuestras responsabilidades intergeneracionales. Ahora, a través de la tecnología digital, la gente se puede expresar libremente, levantar su voz y reclamar no sólo seguridad territorial sino de alimentos, de acceso al agua potable, a los servicios de salud, a la acción rápida, coordinada y eficaz en situaciones de emergencia. Este, y no otro, es el tipo de seguridad que los seres humanos sueñan y merecen.

Palabras clave: Desarme, desarrollo, gasto militar, paz, seguridad.

\begin{abstract}
Federico Mayor Zaragoza affirms in the present text that it is morally unacceptable that every day thousands of people die of hunger when millions of dollars are spent on military and weapons expenditures. He warns that disarmament, including the nuclear, is the best solution and it is reaching points of no return on key issues of intergenerational legacy.

He notes the urgency of a new concept of security, given that the great powers keep thinking that military force is the only expression and reference of "security." The solutions exist. Immediate action is required to fulfill our intergenerational responsibilities. Now, through digital technology, people can freely express, raise their voice and claim not only territorial security but food, access to drinking water, health services, the quick action, coordinated and effective action in emergency situations.
\end{abstract}

Key words: Disarmament, development, military spending, peace, security.

Querida Ingeborg, querida Lisa, queridas amigas y amigos del International Peace Bureau, Reiner, Colin,... Muchas gracias por la organización de este congreso tan importante.

Gracias a todos porque están ustedes promoviendo una movilización en favor de los derechos humanos y de una vida digna para todos los seres humanos.

Gracias en especial a los que han tenido excelentes intervenciones en esta sesión plenaria.

1 Discurso ofrecido en el Congreso Mundial sobre los Gastos Militares y Sociales, organizado por la International Peace Bureau (IPB), realizado del 30 de septiembre al 01 de octubre de 2016, en la Universidad Técnica de Berlín (Sesión Plenaria del 1 de octubre).

2 Presidente de la Fundación Cultura de Paz, Director General de la UNESCO (1987-1999). 


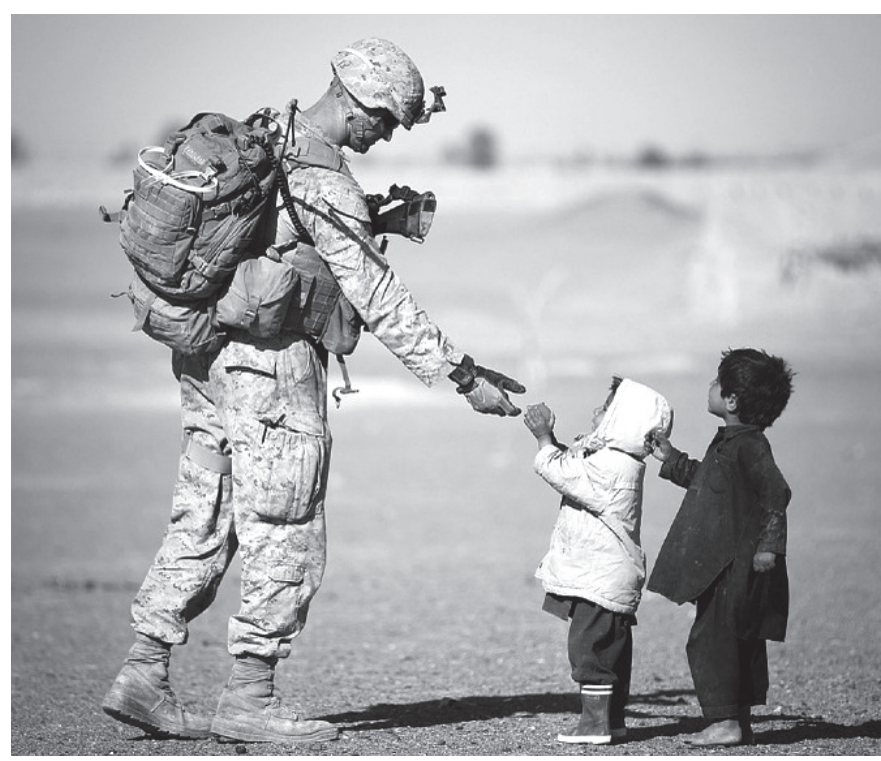

Pixabay.com señores:

Queridos colegas, amigas y amigos, señoras y

Es moralmente inaceptable que cada día mueran de hambre más de 20.000 personas al tiempo que se invierten en gastos militares y armamento 3.000 millones de dólares. Bastaría con una reducción razonable de estas ingentes y desproporcionadas cifras para que pudieran incrementarse rápida y sustancialmente las ayudas al desarrollo endógeno, sostenible y humano, en todo el mundo; se atendiera el crucial legado intergeneracional del medio ambiente, asegurando que no tenga lugar el deterioro irreversible de la habitabilidad de la Tierra; la cooperación internacional permitiría la puesta en práctica de las grandes prioridades de las Naciones Unidas (alimentación, agua, salud, ecología, educación, paz...); $\mathrm{y}$, sobre todo, se haría posible el "nuevo comienzo" que preconiza la Carta de la Tierra.

Es tiempo de acción, porque pueden alcanzarse puntos de no retorno. Ya disponemos de múltiples diagnósticos. Ahora corresponde aplicar sin demora tratamientos adecuados y a tiempo. Los "principios democráticos" - como se define con tanta exactitud y visión en la Constitución de la UNESCO "para guiar a la humanidad" - deben ser incorporados como referencia esencial personal y colectiva en el comportamiento cotidiano y en el marco político.

La nueva era de paz surgirá principalmente de la transición de la fuerza a la palabra, de una cultura de imposición, dominación y violencia a una cultura de encuentro, diálogo, conciliación y paz. En lugar de "si vis pacem para bellum", "si vis pacem para verbum". Mañana puede ser demasiado tarde. Las políticas y estrategias tan bien establecidas en la Declaración y Plan de Acción para una Cultura de Paz de las Naciones Unidas, 1999, deberán aplicarse sin demora.

Es por ello que felicito al IPB porque, conjuntamente con “ ¡Desarme!" han agregado "creando un Plan de Acción". Sí, en la actualidad tenemos muchos diagnósticos y ahora debemos poner en práctica -antes de que sea demasiado tarde- los tratamientos necesarios.

Colin, Reiner, Ingeborg, Lisa... Ésta es la manera. Apenas hace una mes, en las Naciones Unidas, en Nueva York, en el marco del V Foro de Alto Nivel para una Cultura de Paz, Ingeborg Breines intervino con un vibrante discurso sobre "Desarme para el desarrollo" que terminaba así: "En Berlín debemos escribir un plan de acción nuevo, con igualdad de género, no militarista, social". Esto es exactamente lo que necesitamos ahora: un programa de acción que, en el contexto actual, sea capaz de movilizar millones de apoyos en todos los rincones del mundo: “iDesarme para el desarrollo!".

Es preciso advertir que si no hay evolución habrá revolución y que la diferencia entre estas dos palabras es la " $r$ " de responsabilidad. Dejemos de seguir a los irresponsables y, con urgencia, facilitemos la transición de una economía basada en la especulación, la deslocalización productiva y la guerra a una economía de desarrollo sostenible y humano, liderada por un multilateralismo democrático y eficiente.

El neoliberalismo marginó, en los años ochenta del siglo pasado, el Sistema de las Naciones Unidas sustituyéndolo por grupos plutocráticos (G7, G8, G20). ¿Cómo y por qué los países occidentales han aceptado dejar la gobernanza del mundo en tan pocas manos? ¡Somos 193 países!

La mejor solución -aunque tengamos que sobreponernos a la inmensa inercia de quienes se aferran al perverso adagio de "si quieres la paz, prepara la guerra" - es el desarme (incluido, desde luego, el nuclear), aplicando una parte razonable de los colosales medios 
dedicados a la seguridad al desarrollo de todos los pueblos, de tal modo que se haga realidad la igual dignidad y calidad de vida en todos ellos.

\section{El Presidente de las} Conferencias Pugwash, en su excelente intervención, ha subrayado la necesidad de la eliminación urgente de las armas nucleares. En este sentido, considero importante recordar lo que le dijo el Presidente Eisenhower al recién elegido Presidente John F. Kennedy el 20 de enero de 1961, en el traspaso de la presidencia de los Estados Unidos: "Sólo el complejo militar industrial de los Estados Unidos es más poderoso que el Presidente de América del Norte".

En octubre de 1986, estaba yo muy cerca del Presidente Mikhail Gorbachev cuando mantuvo, en Reikiavik, con el Presidente Reagan las reuniones para hacer frente a la amenaza nuclear. En la primera jornada de estas conversaciones estuvieron de acuerdo en reducir un $70 \%$ de las armas nucleares en todo el mundo. Mikhail Gorbachev dijo entonces: “iAhora podríamos destruir el planeta sólo 300 veces!", y le pidió al Presidente Reagan reconsiderar una eliminación completa de las ojivas nucleares. Al día siguiente, cuando el Presidente Reagan mantuvo su posición con firmeza, Mikhail Gorbachev declaró: "Nada ha cambiado en este sentido: como anticipó el Presidente Eisenhower a principios de los años 60, el Partido Republicano insiste en mantener un consorcio militar industrial que es más poderoso que el Presidente de los Estados Unidos"...

Con sólo que se redujeran un $10 \%$ por año y país los fondos invertidos en defensa y en el sector del armamento, la seguridad mundial actual seguiría sin verse afectada, mientras que las principales prioridades de las Naciones Unidas poner en práctica de inmediato, antes de que sea demasiado tarde, ya que estamos ante procesos potencialmente irreversibles. Hay que destacar que tal estrategia de desarrollo conllevaría una mejora general de la economía a escala mundial, contribuyendo a hacer frente a la migración severa y a los problemas sociales que enfrentamos en la actualidad.
¡Es urgente un nuevo concepto de seguridad! Los grandes poderes siguen pensando que la fuerza militar es la única expresión y referencia de "seguridad". Gravísimo error, costosísimo error que se ocupa exclusivamente de los aspectos bélicos y deja totalmente desasistidos otros múltiples aspectos de la seguridad "humana", que es, en cualquier caso, la que realmente interesa.

Cuando observamos los arsenales colmados de cohetes, bombas, aviones y barcos de guerra, submarinos... y volvemos la vista hacia los miles de seres humanos que mueren de hambre cada día, y hacia los que viven en condiciones de extrema pobreza sin acceso a los servicios de salud adecuados... y contemplamos consternados el deterioro progresivo de las condiciones de habitabilidad de la Tierra, nos damos cuenta de que debemos reaccionar sin dilación porque se está llegando a puntos de no retorno en cuestiones esenciales del legado intergeneracional.

El inmenso poder mediático sin precedentes nos convierte en espectadores pasivos en lugar de actores activos. Ahora, gracias a la tecnología digital, la gente se puede expresar libremente, levantar su voz y reclamar no sólo seguridad territorial sino de alimentos, de acceso al agua potable, a los servicios de salud, a la acción rápida, coordinada y eficaz en situaciones de emergencia... Este, y no otro, es el tipo de seguridad que los seres humanos sueñan y merecen.

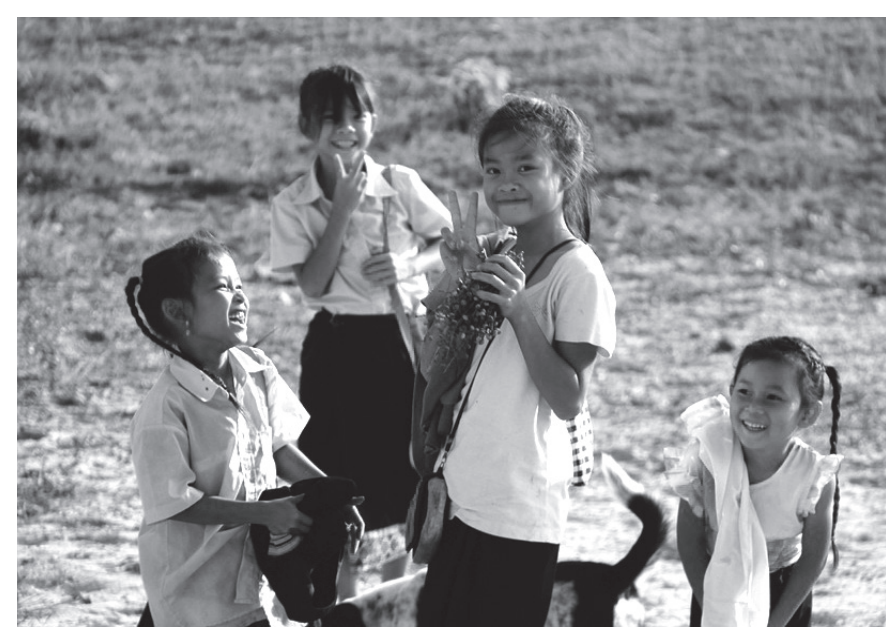

Pixabay.com 


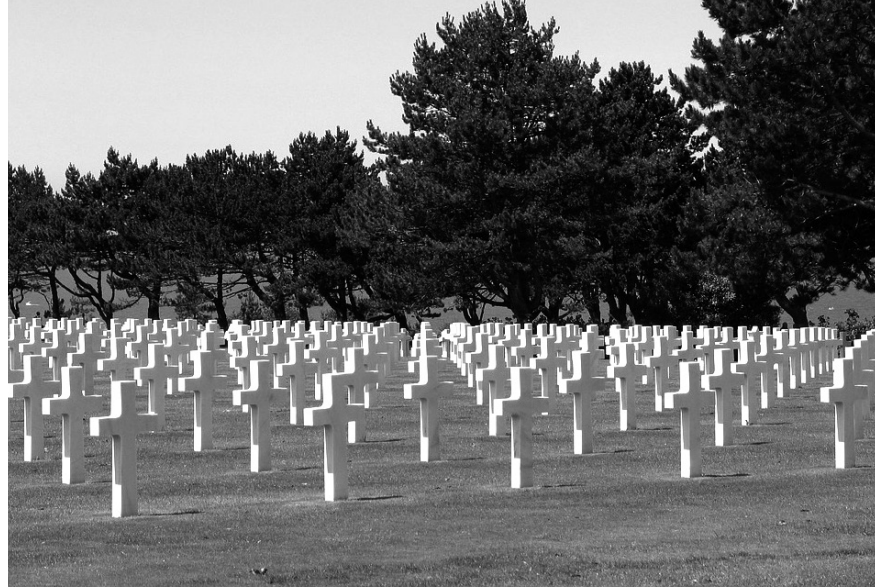

Pixabay.com

Tal como ha declarado el Secretario General Ban-Ki-Moon, "el mundo está demasiado armado y la paz está insuficientemente financiada... Creemos que la transformación de la sociedad sólo puede lograrse manejando los conflictos de forma diferente y reasignando gastos militares". Fondos para poner en práctica el Programa de Acción para una Cultura de Paz, aprobado por la Asamblea General de las Naciones Unidas en septiembre de 1999 y -son muy similares- los objetivos de desarrollo sostenible y las medidas para regular oportunamente el cambio climático. Desarme para el desarrollo: así de sencillo. Las soluciones existen. La acción inmediata es necesaria para cumplir con nuestras responsabilidades intergeneracionales. Es urgente la refundación de las Naciones Unidas.

Ahora ya sabemos. Ahora los seres humanos pueden expresarse y levantar la voz. Ahora, particularmente, las mujeres están aumentando rápidamente su participación en los procesos de toma de decisiones. Me gusta repetir lo que el Presidente Nelson Mandela me dijo en Pretoria en 1996: "Las mujeres son la piedra angular de la nueva era. Ellas serán las protagonistas de la cultura de la paz porque las mujeres utilizan la fuerza sólo excepcionalmente mientras que los hombres sólo excepcionalmente no la utilizan".

Sigamos la iniciativa del International Peace Bureau sobre "Desarme para el desarrollo" y elevemos presencialmente y en el ciberespacio innumerables voces para la justicia y la paz. Una colosal movilización podría iluminar el horizonte sombrío presente. La paz es un derecho... El derecho a la paz está a punto de ser aprobado por la Asamblea General de las Naciones
Unidas. Henning Zierock, un campeón de la cultura de la paz desde 1988, está hoy aquí y nos recuerda que la paz, a través de la justicia, es la mejor manera para superar las amenazas presentes.

Gracias a él, gracias al International Peace Bureau, gracias a todos... Esta transición histórica de la razón de la fuerza a la fuerza de la razón puede tener lugar. Estos sueños pueden convertirse en realidad si aquellos que más han sufrido y los que saben accionar mejor los cambios urgentes que se necesitan, se sitúan en la vanguardia de una movilización masiva, tanto de forma presencial como en el ciberespacio. Ciudadanos del mundo liderados por la comunidad científica, académica, artística, intelectual... ¡Sí!: tener este encuentro en una universidad importante es significativo.

También lo es tener esta reunión en Berlín y transmitir desde aquí nuestros mensajes, es significativo. Caminando "bajo los tilos" (unter der Linden) he escuchado el susurro de "no más guerra, no más guerra"...

De la fuerza a la palabra...

Debemos inventar el futuro. Cada ser humano capaz de crear: esta es nuestra esperanza. Como dijo el Presidente John F. Kennedy en junio de 1963: "No hay ningún desafío que se sitúe más allá de la capacidad creativa distintiva de los seres humanos".

Sí, "Nosotros, los pueblos..." tomaremos en nuestras manos, por fin las riendas de nuestro propio destino.

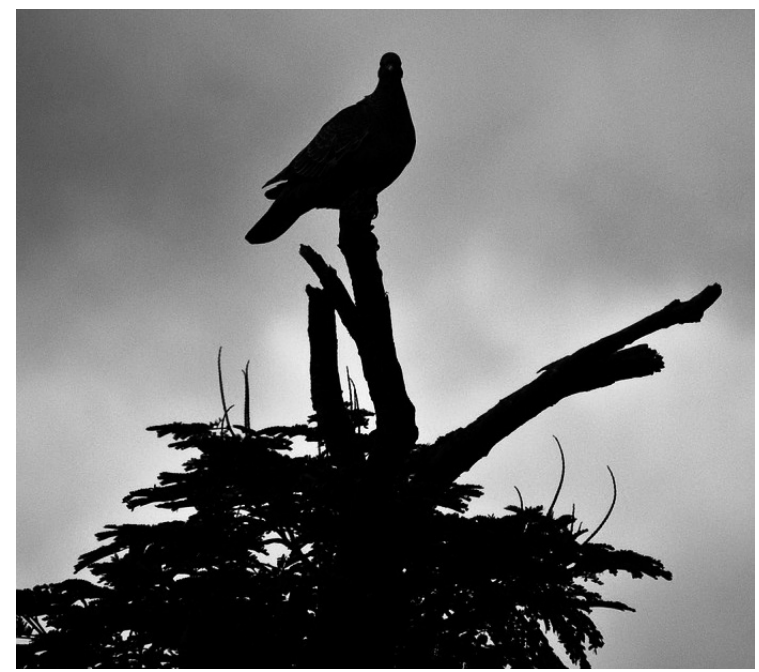

Pixabay.com 